\title{
Pancreatic Society of Great Britain and Ireland
}

The 13th Annual Meeting of the Pancreatic Society of Great Britain and Ireland was held at the Royal Society of Medicine in London on 16 December 1988. The president for that meeting was Professor Tim Northfield and the Lilly Guest Lecturer was Professor Martin Carey of Harvard University Medical College, Boston. The Travelling Fellowship was awarded to Mr C P Armstrong (Bristol) and the Rodney Smith prize for the best paper went to Mr A M Gudgeon (London) for the new work on trypsinogen activation peptide measurement in guaging severity of acute pancreatitis. Selected abstracts are published below.

\section{Caudal drainage after distal resection}

S SHANKAR AND R C G RUSSEll (Department of Gastroenterology, The Middlesex Hospital, London WI) The need for caudal pancreatic drainage after distal pancreatic resection is uncertain. To establish a standard operative technique 88 patients after distal pancreatic resection were analysed comparing two subgroups, one with caudal pancreatojejunostomy Rouxen-y (PJ group) including 33 patients and one without (ND group) including 55 patients.

The median age in years was 36 (ND) and 37 (PJ) and the delay between the onset of disease and operation was 3.5 and 3.8 years respectively. The indication for operation was uncontrolled pain in all. $50 \%$ of patients in the no-drainage group had ductal strictures before resection while $45 \%$ had strictures in the PJ group.

The average duration of the operative procedure in hours was $3 \cdot 2[(2-6 \cdot 5)$ ND] and $3 \cdot 97[(2 \cdot 5-5 \cdot 5) \mathrm{PJ}]$, operative blood loss in litres $1.9(0.45-6.5)$ and $1.6(0 \cdot 1-6)$, and the length of postoperative stay $25(10-84)$ and $31 \cdot 5(10-98)$ days respectively.

Thirty four patients in the ND group and 25 in the PJ were pain free and $82 \%$ of patients in the former and $86 \%$ in the latter required no further hospital admission.

It is concluded that addition of a drainage procedure to a distal pancreatic resection increases the length of the operation and period of postoperative stay without affecting the eventual outcome.

Differential response of human pancreatic cancer to somatostatin and Tamoxifen treatment in vivo

G J POSTON, J P LAWRENCE, P SINGH, C M TOWNSEND JR, AND J C THOMPSON (Department of Surgery, University of Texas

Medical Branch, Galveston, Texas, USA 77550) Recent reports have suggested some reduction in pancreatic cancer growth using the hormone somatostatin and the oestrogen antagonist Tamoxifen. The purpose of these studies was to assess combination therapy with these agents on pancreatic cancers with oestrogen receptors.

Twenty four male nude mice were xenografted in both scapular regions with SKI and a further 24 mice were similarly xenografted with PGER. SKI and PGER were transplantable human pancreatic cancers which possess similar numbers of high affinity, type I oestrogen receptors. Each tumour group of mice were randomised into four equal groups: control; SMS 201-995 $100 \mu \mathrm{g} / \mathrm{kg}$ tds intraperitoneally (long acting somatostatin analogue); Tamoxifen $10 \mathrm{mg}$ / kg every second day subcut; and both agents in combination. Treatment commenced

\begin{tabular}{|c|c|c|c|c|}
\hline & $C O N$ & $S M S$ & $T X N$ & $S M S \& T X N$ \\
\hline \multicolumn{5}{|l|}{ SKI tumour area $\left(\mathrm{mm}^{2}\right)$} \\
\hline Week 2 & $35(2)$ & $22\left(4^{*}\right)$ & $43(6)$ & $42(4)$ \\
\hline Week 4 & $91(8)$ & $53\left(9^{*}\right)$ & $63\left(5^{*}\right)$ & $67\left(8^{*}\right)$ \\
\hline Week 6 & $247(14)$ & $150\left(32^{*}\right)$ & $160\left(13^{*}\right)$ & $157\left(11^{*}\right)$ \\
\hline Week 8 & $388(36)$ & $254\left(47^{*}\right)$ & $294\left(36^{*}\right)$ & $252\left(21^{*}\right)$ \\
\hline \multicolumn{5}{|l|}{ SKI at death } \\
\hline Tumour weight (g) & $2 \cdot 48(0 \cdot 19)$ & $1.71(0 \cdot 36)$ & $2 \cdot 17(0 \cdot 27)$ & $1.71\left(0 \cdot 17^{*}\right)$ \\
\hline Tumour DNA content (mg) & $3 \cdot 60(0 \cdot 50)$ & $1.32\left(0 \cdot 28^{*}\right)$ & $3.09(0.67)$ & $0.95\left(0.21^{*}\right)$ \\
\hline \multicolumn{5}{|l|}{ PGER at death } \\
\hline Tumour weight (g) & $1 \cdot 34(0 \cdot 20)$ & $1 \cdot 31(0 \cdot 22)$ & $1 \cdot 18(0 \cdot 24)$ & $1.41(0.44)$ \\
\hline Tumour DNA content (mg) & $2 \cdot 20(0 \cdot 65)$ & $1.55(0 \cdot 41)$ & $0.70\left(0 \cdot 13^{*}\right)$ & $1 \cdot 30(0 \cdot 19)$ \\
\hline
\end{tabular}

*Significantly $(\mathrm{p}<0 \cdot 05)$ less than control by analysis of variance, confirmed by Students $t$ test.

Differentiation of pancreatic and bile duct cancer from chronic pancreatitis and sclerosing cholangitis using a radiolabelled monoclonal anti-CEA antibody (11-285-14)

A JEWKES, F MACDONALD, W H ALLUM, R DOWNING, AND J NEOPTOLEMOS (Surgical Immunology Unit and Department of Surgery, University of Birmingham and A1160

immediately and continued to sacrifice eight weeks later. Tumour areas were measured twice weekly and at death both normal pancreas and tumours were excised, weighed and assayed for DNA content as a measure cellularity.

There were no differences in body weight or pancreas size during the study and although treatment groups were smaller than control at no point during the study were there significant differences in PGER tumour areas.

Although in some pancreatic cancers combination therapy with somatostatin and Tamoxifen may be more beneficial than either agent alone; in other cancers the effects of these agents may be counter productive. Somatostatin and Tamoxifen probably act on pancreatic cancer through separate and non complementary pathways. ,

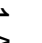

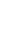


cholangitis using current diagnostic methods. We have recently assessed the accuracy of a new monoclonal anti-CEA antibody (11-285-14) in this context.

First, CEA expression was determined in paraffin sections from cases of pancreatic cancer $(n=30)$, chronic pancreatitis $(n=10)$, cholangiocarcinoma $(n=12)$, and sclerosing cholangitis $(n=4)$. Immunohistochemical staining was assessed by two independent observers. $77 \%$ of pancreatic and $80 \%$ of bile duct cancers were CEA positive. CEA was not detected in sclerosing cholangitis; although $60 \%$ of cases of chronic pancreatitis were positive, staining intensity was markedly reduced.

Because of these encouraging results 25 patients with these conditions were studied in vivo. $200 \mu \mathrm{g}$ of antibody labelled with $0 \cdot 8-1.9 \mathrm{mCi}$ of iodine-131 was given iv and patients imaged at 24 and 48 hours. Diagnosis was confirmed by laparotomy $(n=21)$, CT scanning $(n=1)$, or ERCP $(n=3)$. Eleven out of 12 pancreatic cancers were clearly imaged as were all three biliary tumours. Positive scans were also obtained in $50 \%$ of cases of chronic pancreatitis and sclerosing cholangitis.

Despite the encouraging findings of immunohistochemistry, radioimmunolocalisation using this anti-CEA antibody was unable to reliably differentiate between benign and malignant pancreatic or biliary disease. Antibodies to other tumour associated antigens may prove more useful.

Management of early or late failure of
cholecystojejunostomy for malignant
biliary obstruction by endoscopic endo-
prosthesis insertion

J $F$ DOWSETt, A $R$ W HATFIELD, D VAIRA, C AINLEY, $V$ A CHANDIRAMANI, AND R C G RUSSELL (Departments of Gastroenterology and Surgery, The Middlesex Hospital, Mortimer Street, London) There are now a wide variety of surgical and non-surgical procedures available for the palliation of malignant low biliary obstruction. Each has well recognised advantages and disadvantages. Cholecystojejunostomy is the quickest and easiest surgical alternative when the gall bladder is dilated but its application is limited by an appreciable incidence of early and late malignant cystic duct occlusion. Failure has traditionally meant reoperation. Endoscopic endoprosthesis insertion, however, offers an alternative.

During the last three years, 16 consecutive patients with pancreatic carcinoma presented with malignant biliary obstruction after cholecystojejunostomy. In three, the failure was early with no relief of cholestasis after operation and in 13 the failure was late after initial successful biliary drainage. The median time since surgery in the late failure group was nine mo (range 3-24). Patient parameters (mean/all 16) included age 62 yr [(range 43-79), bilirubin 329 $\mu \mathrm{mol} / \mathrm{l}$ (57-611), albumin $32 \mathrm{~g} / \mathrm{l}$, urea $4 \cdot 3$ $\mathrm{mmol} / \mathrm{l}$, and haemoglobin $10 \cdot 3 \mathrm{~g} / \mathrm{l}]$. The gall bladder was air filled in all cases. All stents placed were $10 \mathrm{Fr}$.

Endoscopic stenting was successful de novo in 11 patients $(69 \%)$. Of the five failures, three had attempted combined percutaneous-endoscopic procedures [one successful and two failed because of inability to pass the stricture percutaneously with a guide wire (external drainage left)], one had a percutaneous endoprosthesis placed because asymptomatic duodenal stenosis prevented endoscopic access and one patient deteriorated rapidly and only had a single endoscopic attempt. Biliary drainage was thus obtained non-surgically in $15 / 16(94 \%)$. Two complications occurred $(12 \cdot 5 \%)$ : cholangitis after failed ERCP drainage and bile leakage after a percutaneous liver puncture. The 30 day mortality rate was $12.5 \%$ (two pts). There were no procedure related deaths. Median survival after stenting was 3.5 mo (range $0 \cdot 5-16)$. There were three late stent changes.

Endoprosthesis insertion is a safe, successful alternative to reoperation for early or late failure of cholecystojcjunostomy for low malignant biliary obstruction. It should be used as soon as surgical failure is recognised.

Endoscopic management of malignant low biliary obstruction caused by unresectable primary pancreatobiliary neoplasm

J $F$ DOWSETT, A $R$ W HATFIELD, $V$ A CHANDIRAMANI, D VAIRA, C AINLEY, AND R C G RUSSELL (Departments of Gastroenterology and Surgery, The Middlesex Hospital, Mortimer Street, London Palliation of malignant low common bile duct obstruction may be achieved by surgical bypass or by endoprosthesis insertion (percutaneous or endoscopic). If symptomatic duodenal stenosis is present and the patient is fit for surgery, triple bypass is the definitive option. If, however, the duodenum is not stenosed and/or the patient is not fit for surgery, endoprosthesis insertion has been proven in prospective trials (Bornman et al Lancet, 1986; i: Dowsett et al Gut 1988, in press) to be as efficacious as surgery. Endoscopic stenting has been shown to have fewer complications than percutaneous stenting (Speer et al Lancet 1987; ii). The prevalence of late stent change and late surgery for duodenal stenosis remains unclear, however.

During the four and a half years at the Middlesex Hospital and three years at the London Hospital up to April 1988, 374 patients with unresectable primary malignant low common bile duct obstruction were treated by endoscopic stent insertion or sphincterotomy (Spx). The latter was used only in nonfriable, true ampullary tumours (11 pts). There were 37 ampullary (A), 321 pancreatic (P), and 16 cholangiocarcinomas $(\mathrm{C})$. The latter were diagnosed if a low biliary stricture was associated with a normal retrograde pancreatogram. Complete follow up is available on $94 \%$. The success rates of drainage were A- $-97 \%$, $\mathrm{P}-84 \%$, and $\mathrm{C}-75 \%$ and the procedural complication rates were $32 \%$ (Spx $36 \%$; stent $30 \%), 19 \%$, and $19 \%$ respectively. The procedure related and 30 day mortality rates were $\mathrm{A}-3 \% / 3 \%, \mathrm{P}-2 \% / 19 \%$, and C- $6 \% / 44 \%$. The median survivals were nine months, 4.5 months, and two months respectively. The number of late stent (re)placements was A-14 (12 pts $=32 \%$; $9=$ initial stent, $3=$ initial Spx), P-92 (49 pts= $12 \cdot 5 \%)$, and $\mathrm{C}-1(1 \mathrm{pt}=6 \%)$. Late surgery because of symptomatic duodenal stenosis was required in $10 \cdot 8,3 \cdot 1 \%$, and $0 \%$ respectively.

Endoscopic management of low primary malignant biliary obstruction due unresectable tumours is successful and has an acceptable carly and late complication rate.

Efficacy of acid resistant fungal lipase in steatorrhoea as a result of adult cystic fibrosis

P ZENTLER-MUNRO, B A ASSOUFI, S CORNNELL, M E HODSON, AND T C NORTHFIELD (Department of Cystic Fibrosis, Brompton Hospital and Department of Medicine, St George's Hospital Medical School, London) Pancreatic steatorrhoea is often resistant to high doses of porcine pancreatin, because the enzymes are destroyed by acid in the stomach. We have recently shown that an enzyme prepared from Aspergillus niger retains activity within the stomach of $\mathrm{CF}$ patients. We have therefore compared this acid resistant fungal lipase with two preparations of enteric coated microspherules of pancreatin (Creon and Lipagest) in 10 adults with steatorrhoea due to $\mathrm{CF}$. Three consecutive two week treatment periods were preceded by a two week control on no 
treatment. Fat intake was the same for each period. Faecal wet weight (mean (SEM) g/ three days), was 1220 (164) on no treatment. This was reduced to 857 (145) on Creon and 950 (222) on Lipagest $(\mathrm{p}<0 \cdot 01)$; but remained unchanged at 1137 (131) on fungal lipase. Faecal fat excretion ( $\mathrm{g} / 3$ days) was $118 \cdot 3(22 \cdot 7)$ on no treatment. This was reduced to $44.6(7.9)$ on Creon $(\mathrm{p}<0.01)$ and to $76 \cdot 0(19 \cdot 5)$ on Lipagest $(p<0 \cdot 05)$; but was unchanged at $108 \cdot 5(21 \cdot 1)$ on fungal lipase.

We conclude that, whereas both enteric coated pancreatin preparations were efficacious in treatment of pancreatic steatorrhoea, the acid resistant fungal lipase was not. Possible reasons include inhibition of fungal lipase by intraduodenal bile acids, as shown in vitro.

Incidence and mortality from acute pancreatitis in Scotland, 1961-85

C WILSON AND C W IMRIE (Department of Surgery, Royal Infirmary Glasgow, Scotland) Few studies have examined the changing trends in incidence and mortality from acute pancreatitis. Studies from Bristol have shown an increasing incidence over the 30 year period 1950-79 although case mortality has remained unchanged at around $20 \%$ throughout. Data on all hospital admissions in Scotland have been recorded since 1961 as the Scottish Hospital Inpatient Statistics, permitting analysis of these trends. The annual admissions for acute pancreatitis in men have increased 11 fold from 69 cases/year in 1961 to 750 cases/ year in 1985 and in women four-fold from 118 cases/year to 494 cases/year respectively. This increase was seen particularly in young and middle aged adult men (20-59 years) and in elderly women (60 years). Mortality in hospital did not show a corresponding change, increasing only two-fold in men from 15 cases/year to 30 cases/year and in women from 29 cases/year to 37 cases/ year. Case mortality showed a dramatic fall from $18 \%$ in $1961-65$ to $5 \cdot 5 \%$ in $1981-85$. The most marked increases in incidence were recorded within the Health Board area of central Scotland. The largest increase in admissions occurred in the six year period after 1971 and coincided with the introduction of the Phadebas amylase test (Pharmacia). It is suggested that much of the apparent increase in incidence of acute pancreatitis may be attributed to an increased diagnostic rate because of greater clinical awareness and the availability of a simple, reproducible diagnostic test.
New evidence demonstrating impaired fibrinolysis in acute pancreatitis

CHRISTINE HALL, M J WEBBERLEY, $M$ T DONNAN, T LEESE, AND J P NEOPTOLEMOS (Departments of Surgery, Gastroenterology, and Haematology, Dudley Road Hospital, Birmingham and Leicester Royal Infirmary, Leicester) Although certain aspects of the clotting cascade are well described in acute pancreatitis (AP), specific aspects of fibrinolysis are unclear. We studied 29 cases, 21 predicted mild and eight predicted severe (Glasgow system). We confirmed previous studies showing that fibrinogen did not correlate with disease activity and also a decrease in plasminogen activity. We measured intrinsic and extrinsic pathways of fibrinolysis by urokinase and euglobulin lysis time (ELT) tests. In AP, median (range) times for urokinase (mins) were 14 $(9-26), 13(8-27)$, and $15(10-14)$ on days 1 . 2 , and 3 , all significantly different from controls: $10(8 \cdot 5-13), 9$ (8-14), 10 (8-14) respectively (all $\mathrm{p}<0 \cdot 01$, Mann-U test). ELT tests in AP were also all significantly prolonged: 340 (180-900), 220 (165-900), 200 (65-180) on days 1,2 , and 3 compared with controls: $120(60-150), 105(90-135)$, $120(65-180)$ respectively (all $\mathrm{p}<0 \cdot 001)$. Values returned to normal after recovery. A stressed ELT test, however, was found to normalise abnormally high values.

These findings indicate impaired fibrinolysis rather than increased fibrinolysis previously shown. Moreover, the results of the stressed ELT test indicate that adequate circulating tissue plasminogen activator is released but appears to be blocked, probably by an unknown circulating inhibitor.

Acute biliary pancreatitis (ABP): a retrospective analysis of endoscopic approach in 61 cases

J DEUS, A MARQUES, A GINESTAL-CRUZ, M CÉSAR, N GRIMA, AND J PINTO CORREIA (Department of Medicine 2 (UCIGE), University Hospital of Santa Maria, Centre of Gastroenterology (INIC), Lisbon, Portugal) From December 1980 to December 1986, 61 patients (pts) - 18 male, 43 female; mean age 61 years - admitted to our Intensive Care Unit with ABP were submitted to Endoscopic Retrograde Cholangiopancreatography (ERCP), followed in 30 by Endoscopic Sphincterotomy (ES). Fifteen of the 61 pts had previous cholecystectomy. ERCP was performed within 10 days after onset of AP in 28 pts, 20 of which through clinical criteria of urgency (followed by ES in 17). Indications for endoscopic approach in the whole group were: increasing cholestasis (14 pts), acute cholangitis (11), clinical deterioration of ABP (5), pre-surgical evaluation (4), and aetiological confirmation (27). In 57 pts with suspected gall stones from clinical (MQ1 +ve) ${ }^{1}$ and/or echographic criteria. ERC was successful in $54(95 \%)$ and confirmed biliary actiology (stones in 45 , other causes in nine). In the remaining four pts, without clinical (MQ1) or echographic suspicion of gall stones, these have been identified by ERC in three. All 30 pts with ES (13 with $\geqslant$ three Ranson prognostic criteria) had adequate biliary drainage (stone removal in $18 \mathrm{pts}$ ), without induced morbidity. None of ES treated pts has further episodes of ABP although 11 of 17 pts with associated gall bladder lithiasis had no elective cholecystectomy. In one of 11 pts with acute cholangitis emergent ES could not change clinical evolution to sepsis and death.

Endoscopic approach may bring a safe contribution to determine the diagnosis and therapy in ABP and should be prospectively evaluated.

Reference

1 Deus J, Cesar M, Marques A et al. Early diagnosis of biliary aetiology in acute pancreatitis. Gut 1987; 28-3: A370.

Dynamic CT angiography - a technique for precise identification of pancreatic necrosis

M LARVIN, A G CHALMERS, AND M J MCMAHON (University of Surgery and Department of Diagnostic Radiology, The General Infirmary, Leeds) Most patients with acute pancreatitis (AP) are successfully managed by conservative means, but a minority develop pancreatic necrosis requiring surgical intervention. Selection of patients is dependent upon accurate identification of pancreatic and peripancreatic necrosis, for which ultrasound and computed tomography (CT) have proved unreliable.

The role of dynamic CT angiography was investigated in 49 patients with severe AP. Using an IGE-9800 scanner, $10 \mathrm{~mm}$ upper abdominal images were obtained after administration of dilute oral contrast. Targeted $5 \mathrm{~mm}$ images were acquired dynamically (two second scans, eight second cycle) through the pancreas, for one minute after rapid injection of concentrated non-ionic contrast ('Niopam 370', Merck) at a dose of $1.5-2 \mathrm{ml} / \mathrm{kg}(0.5-0.75 \mathrm{~g}$ iodine/ $\mathrm{kg}$ ). Criteria for selection of patients for scanning included established organ-system failure $(n=9)$; increasing APACHE-II 
score (Ref) at five days $(\mathrm{n}=24)$; suspected necrosis on ultrasound or CT prior to referral $(n=16)$. The presence of necrosis was established at laparotomy or autopsy.

Pancreatic or peripancreatic necrosis was present in nine cases, and in each there were one or more areas of non-enhancing pancreatic parenchyma. Pancreatic enhancement was considered normal in 40 patients, all of whom survived without laparotomy to remove necrotic pancreas, although nine were subsequently treated for localised collections of fluid or pus (percutancous aspiration $n=5$, laparotomy and drainage $\mathrm{n}=4$ ). Dynamic CT angiography appears to provide precise diagnosis and localisation of pancreatic necrosis.

Reference

Knaus WA, Draper EA, Wagner DP, Zimmerman JE, APACHE-II: A severity of disease classification system. Crit Care Med 1985: 13: 818-29.

Biochemical studies of peritoneal exudates and pseudocyst fluid

C WILSON, D HEATH, A SHENKIN, AND C W IMRIE (Departments of Surgery and Biochemistry, Royal Infirmary, Glasgow) Acute pancreatitis (AP) exudate appears to be toxic in experimental studies, perhaps because of overwhelming of the antiprotease defences by proteolytic enzyme release. We have studied proteolytic enzyme activation and the adequacy of the antiprotease defences in exudates from human AP (21 patients), intestinal obstruction (eight), perforated peptic ulcer (seven), and in pseudocyst fluid (12).

The three exudates had similar protein and alpha $a_{1}$ antiprotease concentrations. Acute pancreatitis and perforated ulcer exudates frequently showed tryptic amidase activity (indicating trypsin/alpha ${ }_{2}$ macroglobulin complexes) and had reduced trypsin binding capacities compared with intestinal obstruction exudates (67 and 36 $\mu \mathrm{g} / 100 \mu \mathrm{l} v 115 \mu \mathrm{g} / 100 \mu \mathrm{l}, \mathrm{p}<0 \cdot 001)$. Free proteolytic activity (indicating overwhelming of the antiprotease defences) was not seen. Pseudocyst fluids showed a significantly lower mean alpha ${ }_{2}$ macroglobulin and trypsin binding capacity $(11 \mu \mathrm{g} / 100 \mu \mathrm{l})$; but the highest tryptic amidase activities with six fluids showing fresh proteolytic activity. Peritoneal antiprotease defences appeared sufficient for the degree of proteolytic enzyme release in all but two patients with AP, both dying of shock within 24 hours of admission. Antiprotease defences were often insufficient within pseudocysts with resulting uncontrolled proteolytic activity, although, confined to the cyst, this does not appear to pose a threat.

A multicentre controlled clinical trial of high volume fresh frozen plasma therapy in prognostically severe acute pancreatitis

T LEESE, M WATKINS, W M THOMAS, A ATTARD, J P NEOPTOLEMOS, C HALL, AND M HOLLIDAY (Departments of Surgery, Universities of Leicester and Birmingham) An uncontrolled clinical trial' and an animal study' have suggested that fresh frozen plasma (FFP) improves outcome in acute pancreatitis. The proposed mechanism is the replenishment of important plasma proteins, particularly alpha ${ }_{2}$ macroglobulin $\left(\mathrm{A}_{2} \mathrm{M}\right)$. $\mathrm{A}$ controlled trial of low volume FFP therapy failed to show clinical benefit, but confirmed that declining serum $\mathrm{A}_{2} \mathrm{M}$ concentrations can be supplemented during the early stages of the disease. ${ }^{3}$

A further controlled trial of high volume FFP therapy, including only patients with prognostically severe pancreatitis, is underway in four Midlands hospitals. The eight modified Glasgow prognostic criteria ${ }^{+}$are measured within six hours of diagnosis of acute pancreatitis (serum amylase $>1000$ IU/I). Only patients with prognostically severe disease ( $\geqslant 3$ adverse factors) are entered into the trial. They are randomised to receive 8 IU FFP or a similar volume of human albumin solution (colloid control) per 24 hours for 72 hours as part of the intravenous fluid therapy. The major serum antiprotease C-reactive protein, fibronectin, fibrinogen, fibrin degradation products, and D-dimer are measured on days 1,3 , and 7 .

Of 129 patients with acute pancreatitis seen to date, 28 with prognostically severe disease have been randomised into the trial. No significant difference in clinical outcome is apparent between the treatment groups. There have been four deaths in the colloid control group $(40 \%)$ and one in the FFP treated group $(5 \%)$. FFP therapy maintains higher serum $\mathrm{A}_{2} \mathrm{M}$ concentrations on day 3 $(p<0.05)$ and day $7(p<0.05)$ and higher serum antithrombin 111 concentrations on day $3(p<0.05)$ compared with the colloid control group. Fibrinogen concentrations are also higher on day 3 in the FFP treated group $(p<0 \cdot 02)$. We feel that these results justify the continuation of the trial.

\section{References}

1 Cuschicri A, et al. Br J Surg 1983: 70: 710-2.

2 Leese T, et al. Int J Pancreatology 1988. (In press).

3 Leese T, et al. Br J Surg 1987; 74: 907-11.

4 Blamey SL, et al. Gut 1984; 25: 1340-6.
Low level proteinuria: a potential predictor of severity in acute pancreatitis

CLIFFORD P SHEARMAN AND PETER GOSLING (Departments of Surgery and Biochemistry, Selly Oak Hospital, Birmingham) Low level protcinuria undetectable by conventional qualitative testing, occurs in a variety of acute non-renal conditions such as trauma, burns, ischaemia and surgery, in which there is a generalised increase in vascular endothelial permeability in association with acute inflammation. This prompted us to suggest low-level proteinuria may be a sensor of disease severity in acute pancreatitis. We examined urinary albumin and urinary IgG in 19 patients with acute pancreatitis. After admission and diagnosis all urine was saved in six hourly timed collections. Urinary albumin and IgG were measured by sensitive immunoassay; median (range) values for normal subjects were $3.4(0.2-22 \cdot 9)$ and $1.4(0.2-4.5) \mu \mathrm{g} /$ minute respectively.

Sixteen patients made an uneventful recovery (group I), and three patients developed complications (group II), two of which were fatal (respiratory failure, respiratory and renal failure) and one patient survived respiratory failure to develop a pancreatic pseudocyst. Urinary albumin and IgG showed rapid changes which reflected the clinical state in all patients. In group I peak urinary protein excretion occurred during the first $36 \mathrm{~h}$ after admission, and fell rapidly to normal in the subsequent five days. In the non-surviving patients in group II urinary proteins remained raised. The mean (SD) peak albumin and IgG excretion rates in the first 36 hours following admission for group I were $101.4(98.4)$ and $22.0(29.3) \mu \mathrm{g} /$ minute respectively, and for group II 297.9 $(61.2)$ and $106.5(23.8) \mu \mathrm{g} /$ minute respectively $(\mathrm{p}<0 \cdot 01$; Wilcoxon's).

These preliminary data support the hypothesis that low level proteinuria is a very early response in severe inflammatory conditions such as acute pancreatitis, and may be a useful predictor of disease severity.

Assessment and monitoring of acute pancreatitis by the APACHE II scoring system

D J HEATH, C WILSON, AND C W IMRIE (Department of Surgery, Glasgow Royal Infirmary, Glasgow) Multiple factor scoring systems such as those described by Ranson and Imric are accepted means of predicting the outcome for acute pancreatitis. Their drawbacks include a delay of up to 48 hours 
before an answer can be obtained, and a once only prediction which precludes sequential monitoring of the course of the illness. Knaus et al in 1981 described a method of stratifying acutely ill patients; the acute physiology and chronic health evaluation (APACHE) score. This has been modified to include five physiological and seven laboratory parameters as well as a weighting for age and chronic health state (APACHE II). We have prospectively evaluated these parameters in 157 consecutive patients with acute pancreatitis. Thirty five patients had a complicated attack with 12 deaths, the remainder being uncomplicated.

The APACHE II score distinguished between fatal and uncomplicated, and complicated and uncomplicated attacks on all days (6.4 $v$ 12.4 $\mathrm{p}>0.0001$ MannWhitney U test). Using a peak APACHE II score of $>9$ as a discriminating value, we compared sensitivity, specificity and percentage correct with the Ranson and Imrie scoring systems. The first day APACHE II had a sensitivity and a specificity of $66 \%$ and thus acted as an indicator of disease severity within a few hours of admission. Comparison of the mean daily APACHE II scores between days 1 and 3 showed a highly significant fall in those patients with mild disease (Wilcoxon's rank-sum test; $\mathrm{p}<0.0001$ ) a slight but non significant rise in those with a fatal outcome.

The APACHE II system compares favourably with the existing scoring systems in assessing the outcome in acute pancreatitis. It has the additional advantage of being available within a few hours of admission and can be used in monitoring the disease course.

\section{Plasma fibronectin and acute phase protein fluxes during acute pancreatitis}

M LARVIN, G F YOUNG, AND M J MCMAHON (University Department of Surgery and Renal Research Unit, Leeds General Infirmary, Leeds) Fibronectin (FN) opsonises noxious bacterial and particulate material, enhancing their adhesion to phagocytes. Decreased plasma FN levels are associated with poor prognosis in septi-

caemia, burns, and severe trauma, as are increased concentrations of acute phase proteins. ' The aim of the study was to investigate the relationship between fluxes in plasma $\mathrm{FN}$, acute phase proteins, and the severity of acute pancreatitis (AP). Plasma FN, C-reactive protein (CRP), alpha-I-acid glycoprotein (AAG), prealbumin (PA), and transferrin (TF) were measured using an automated immunochemical analyser, in samples obtained on days $1,3,5$, and 7 of 20 attacks of AP. Twelve 'mild' attacks were uncomplicated, whilst eight 'severe' attacks were complicated by organ system failure $(n=6)$ and/or pancreatic necrosis $(n=2)$.

In severe attacks, FN concentrations fell significantly more, CRP and AAG values rose significantly, whilst $\mathrm{PA}$ and TF levels

\begin{tabular}{lllcc}
\hline & & FN mg/l & CRP $m g / l$ & AAG mg/dl \\
\hline \multirow{2}{*}{ Day 1 } & Mild & $224(163-314)$ & $6(6-97)$ & $80(27-109)$ \\
& Severe & $182(107-278)$ & $96(6-239)^{*}$ & $72(51-165)^{\dagger}$ \\
Day 3 & Mild & $203(153-261)$ & $80(27-174)$ & $79(56-106)$ \\
& Severe & $134(75-171)^{*}$ & $193(109-319)^{*}$ & $114(69-150)^{*}$ \\
Day 5 & Mild & $179(113-276)$ & $111(20-157)$ & $97(72-137)$ \\
& Severe & $113(54-181)^{*}$ & $156(108-242)^{\dagger}$ & $129(89-182)^{\dagger}$ \\
Day 7 & Mild & $199(140-291)$ & $47(6-107)$ & $104(69-147)$ \\
& Severe & $151(43-219)^{\dagger}$ & $84(23-163)$ & $139(89-195)^{\dagger}$ \\
\hline
\end{tabular}

(Median values and ranges): ${ }^{*} \mathrm{p}<0 \cdot(22: \dagger \mathrm{p}<0.05$.

Clinical trial of trypsinogen activation peptide (TIP) assay in severity prediction of acute pancreatitis

A M GUDGEON, D HEATH, P HURLEY, A SHENKIN, A JEHANLI, G PATEL, C WILSON, B AUSTEN, C W IMRIE, AND J HERMON-TAYLOR (Departments of Surgery, St George's Hospital Medical School, London and Royal Infirmary, Glasgow) Acute pancreatitis lacks a simple specific test for early severity prediction. We have developed a sensitive immunoassay specific for free TAP (J Immunol Meth 1988, III; 195-203) released on trypsinogen activation. Simultaneous samples of serum and urine were obtained on admission and at intervals from 53 patients with acute pancreatitis in whom the first symptom to sampling time was 48 hours or less. Serum was assayed for Creactive protein (CRP) and amylase, and urine for TAP using RIA.

Patients were allocated to mild or severe groups retrospectively. The mild group con- declined. There were significant correlations between plasma FN and CRP $(p=0 \cdot 6)$, AAG $(p=0.51)$, PA $(p=0 \cdot 59)$, and TF $(\mathrm{p}=0.57)$ values (Spearman test, all $p<0 \cdot 001)$. Thus, it may be possible to identify patients with plasma FN deficiency more rapidly by measuring CRP. The cause of falling plasma FN levels during AP is unknown, but the association with the acute phase protein response suggests some degree of common control.

\section{References}

1 Wilson C. Maharaj D, Gemmell C. Imrie CW. Can fibronectin predict the outcome of acute pancreatitis? Br J Surg 1988; 75.

2 Mayer AD, McMahon MJ, Bowen M, Cooper EM. C-reactive protein: an aid to the assessment and monitoring of acute pancreatitis. J Clin Pathol 1984: 37: 207-11

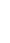

